\title{
Investigations of Sawtooth Oscillations with the Greifswald EBIT
}

\author{
Birgit Schabinger*, Christoph Biedermann ${ }^{\dagger}$, Stephan Gierke*, \\ Gerrit Marx*, Rainer Radtke ${ }^{\dagger, *}$ and Lutz Schweikhard* \\ *Ernst Moritz Arndt University of Greifswald, Institute of Physics, Felix-Hausdorff-Str. 6, \\ 17489 Greifswald, Germany \\ ${ }^{\dagger}$ Max Planck Institute for Plasma Physics, EURATOM Association, Wendelsteinstr. 1, \\ 17491 Greifswald, Germany
}

\begin{abstract}
The former Berlin electron-beam ion-trap was moved to Greifswald. One of the first aims after the reinstallation was the continuation of experiments using mixed ensembles of lowand high- $Z$ ions for further studies of the previously reported sawtooth-like oscillations of the trap plasma. First results of these studies for xenon/argon mixtures are presented.
\end{abstract}

Keywords: EBIT; highly charged ions; confinement; evaporative cooling

PACS: 34 .80.Dp, 52.58.Qv, 32.30.Rj, 34.80.Lx

\section{INTRODUCTION}

Electron-beam ion-traps (EBITs) (Fig. 1) allow the production and storage of highly charged ions (HCIs) $[1,2,3]$, with charge states up to $q=92+$ in the case of uranium [4]. To this end, a mono-energetic electron beam launched from a indirectly heated cathode is compressed by the magnetic field of two Helmholtz coils, thus producing an electron density of several thousands of $\mathrm{A} / \mathrm{cm}^{2}$. After passing three drift tubes the electron beam is caught by a collector. The center drift tube has a larger diameter than the outer electrodes (Fig. 1). By this choice of geometry the image charge of the electron beam provides an axial trapping potential for the confined ions. This potential can be further increase by applying bias voltage to the end drift tubes. The radial trapping potential is created by the space charge of the electron beam.

Neutral atoms or low-charged ions are injected into the EBIT and ionized by electron impact. The ions' charge-state distribution is given by the energy of the electron beam across the ionization potentials of subsequent ionization stages. In order to avoid chargeexchange processes by interaction with neutral residual-gas particles, which would shift the charge distribution to lower states, ultrahigh-vacuum conditions are required.

In addition, the HCIs can be extracted from the EBIT. This will allow us to investigate collisions between HCIs and atomic clusters. It is planned to study charge exchange and fragmentation processes [5]. For reactions between neutral fullerenes and, e.g. singly charged ions low charge fullerenes up to $\mathrm{C}_{60}^{5+}$, smaller $\mathrm{C}_{60-2 m}^{q+}$ fullerenes and small singly charged fragments between $\mathrm{C}_{1}^{+}$and $\mathrm{C}_{29}^{+}$were reported [6]. In contrast, the use of highly charged ions led to charged fullerenes of up to $\mathrm{C}_{60}^{9+}$ [7]. We propose to extend these measurements by varying the kinetic energy and the charge state of the ions. After 


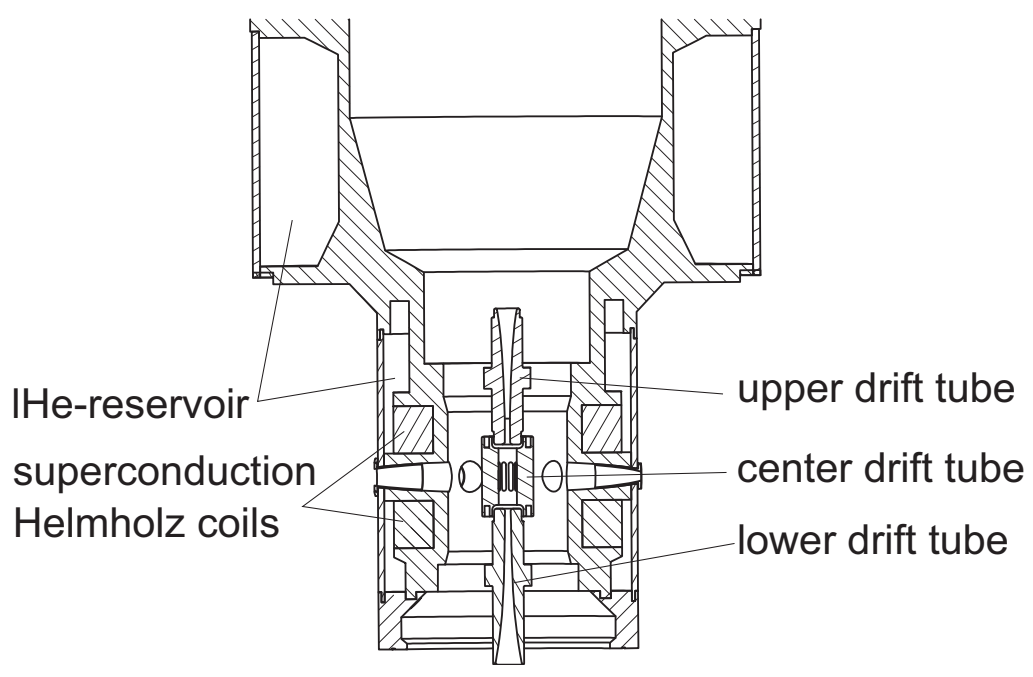

FIGURE 1. Vertical cross section of the Greifswald EBIT.

initial tests with fullerenes, the experiments in Greifswald are planned to be continued with cooled metal clusters.

Our first measurements at the EBIT's new location, however, were performed with mixed gas ensembles in a shallow trap with an axial potential varying from a few volts to several tens of volts. Under these conditions sawtooth-like oscillations of the emitted X-rays from argon and barium ions were observed for the first time in 2003 at the Berlin EBIT [8]. In addition, experiments with xenon and argon or krypton ions were performed [9]. Furthermore, oscillations of the x-ray emission of xenon and krypton ions were also observed for a deep trapping potential of $300 \mathrm{~V}$ at the Shanghai EBIT [10]. However these $\mathrm{Xe}$ and $\mathrm{Kr}$ oscillations appeared synchronously, in contrast to the oscillations observed at the Berlin EBIT, where the oscillations of the heavier and the lighter ion species had opposite phases.

In the following, the experimental setup is described and a short introduction into the sawtooth mechanism is given. First results from the Greifswald EBIT and the simulation of the sawtooth oscillation are presented.

\section{EXPERIMENTAL SETUP}

Compared to its former location at Berlin no modification of the EBIT (Fig. 1) itself was introduced during its reassembling at Greifswald. The detailed parameters are given in Tab. 1 for the former Berlin EBIT $[1,11]$ - now Greifswald EBIT.

The two superconducting Helmholtz coils produce a magnetic-flux density of $3 \mathrm{~T}$. Liquid helium is necessary for operation of the coils and also provides the cooling of the vacuum vessel to guarantee the required vacuum conditions.

The center drift tube has eight slots allowing, e.g., neutral-gas injection and x-ray spectroscopic measurements of HCIs using a windowless germanium detector [11] sensitive in the energy range above $500 \mathrm{eV}$. A cross section of the EBIT's center with the solid-state detector and the gas-injection system is shown in Fig. 2. 
TABLE 1. Physical parameters of the Greifswald EBIT $[11,1]$.

\begin{tabular}{lc}
\hline number of drift tubes & 3 \\
center-drift tube: length & $4 \mathrm{~cm}$ \\
center-drift tube: diameter & $1 \mathrm{~cm}$ \\
end-drift tubes: length & $9 \mathrm{~cm}$ \\
end-drift tubes: diameter & $0.3 \mathrm{~cm}$ (next to the center drift tube) \\
& $1.4 \mathrm{~cm}$ (outer end) \\
magnet-flux density & $3 \mathrm{~T}$ \\
electron gun: & tungsten; $3 \mathrm{~mm}$ diam. \\
max. electron-beam current & $150 \mathrm{~mA}$ \\
max. electron density & $5 \times 10^{12} \mathrm{~cm}^{2}$ \\
diameter of electron beam & $70 \mu \mathrm{m}$ \\
max. electron-beam energy & $30 \mathrm{keV}$ \\
highest ion charge state & He-like W72+ \\
\hline
\end{tabular}

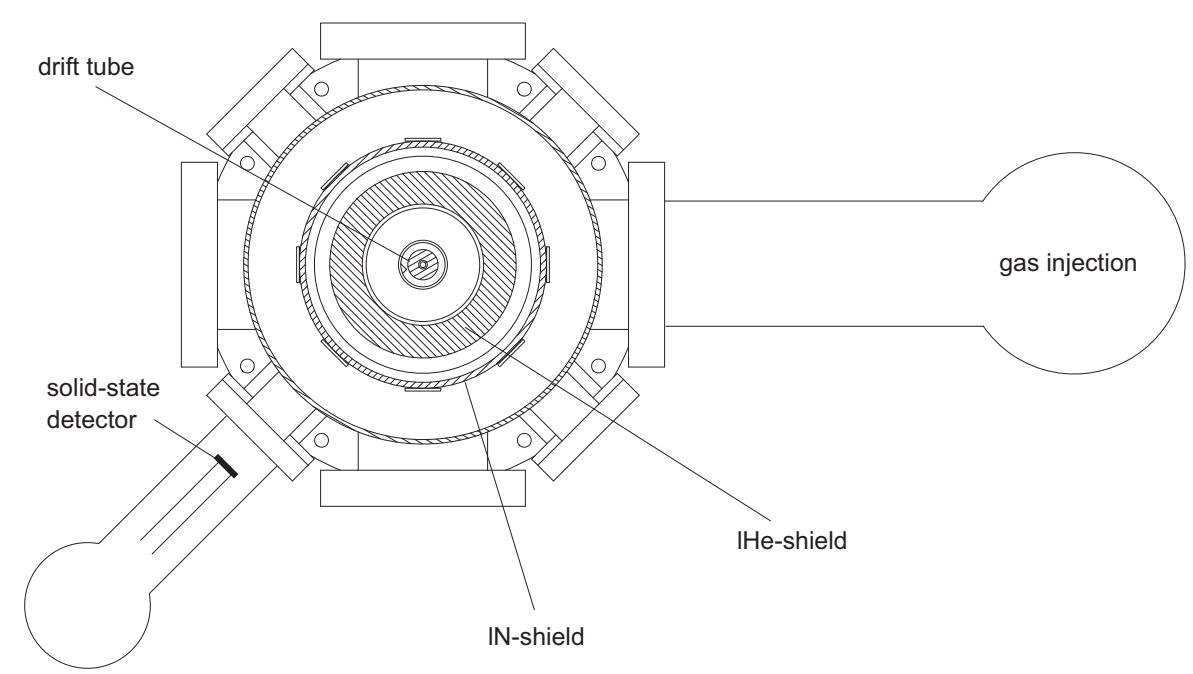

FIGURE 2. Horizontal cross section of the EBIT with layout of the solid-state detector and the gasinjection system.

\section{EVAPORATIVE COOLING AND SAWTOOTH OSCILLATIONS}

As described above, neutral atoms or low-charge ions injected into the EBIT are ionized by multiple electron impact and confined by the axial and radial trapping potentials. In addition, the colliding electrons also heat the ions [12]. As a result of the energy gain, some ions escape from the trap.

To reduce this outflow and increase the trapping lifetimes for confined ions, the experiment can be performed with a mixture of different ion species and thus charge states. When, in addition to a heavy component, a small portion of a light element is injected into the trap, energy is transferred between the higher charge-state ions and the light ions due to the ion-ion collisions. Since the light and thus less highly charged ions are bound weaker by the trapping potential they are lost by axial escape. In this way energy is removed from the ion ensemble and, accordingly, this technique is termed 


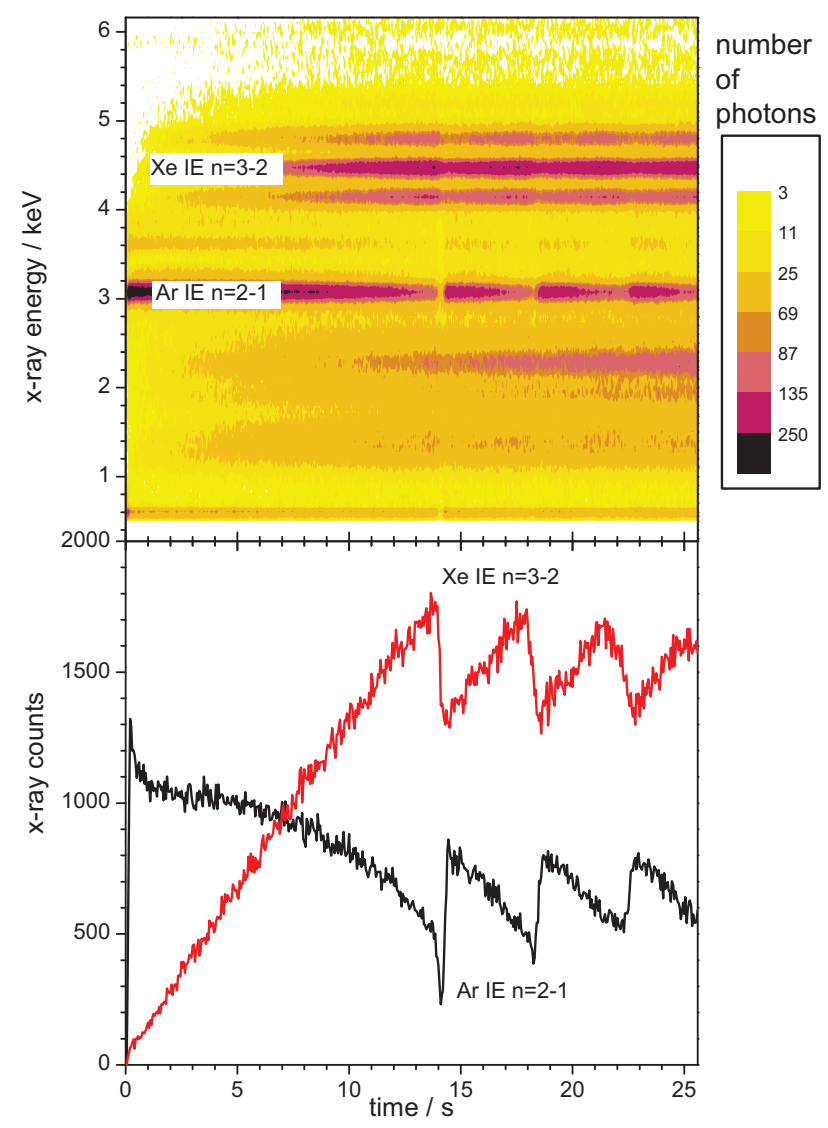

FIGURE 3. (Top) X-ray emission of the trap content versus time at $40 \mathrm{~mA}$ electron-beam current, $5 \mathrm{keV}$ electron-beam energy and a bias voltage of $10 \mathrm{~V}$ between center and upper drift tube. The emission bands from impact excitation (IE) of $\operatorname{Ar}^{16+}$ ( $n=2-1$ transition) and $\mathrm{Xe}^{44+}(n=3-2$ transition) are marked. (Bottom) Projection of the emission intensity in the bands onto the time axis. The x-ray emissions from argon and xenon show opposite trends in the sawtooth oscillations.

evaporative cooling. Evaporative cooling is the key to the successful operation of an EBIT and permits the production and storage of a much higher number of highly charged ions than would be possible without cooling [13].

Of course, the evolution of ions in an EBIT depends on the operating and device parameters (electron-beam current and energy, axial potential wall) and the rates at which neutral atoms are fed to the EBIT. If the commonly applied conditions for the evaporative-cooling technique are changed and much more light than heavy gas is injected into the trap, a sawtooth activity is observed. This sawtooth activity of the trap content was reported for the first time form the Berlin EBIT [8]. Figure 3 shows the results from a recent measurement at the Greifswald EBIT for a system of highly charged Ar and Xe ions: X-rays from electron-impact excitation are presented. A prominent feature is the mirror-like behavior of the intensities for $\mathrm{Ar}$ and $\mathrm{Xe}$. The population in the trap does not reach a steady state. Instead, Ar ions are continuously replaced by Xe ions until a substantial part of ions are lost from the trap by rapid axial 
expulsion [9]. The sawtooth activity may be explained along the following line: Xe ions accumulate in the trap; they are cooled by the Ar ions which in turn escape from the trap more rapidly because of their lower charge. This process continues until a situation is approached where the cooling is much reduced because of insufficient Ar density. As a consequence, the Xe ions encounter substantially more heating. As the ion temperatures rise the particle loss is further enhanced (ion expulsion). The lower charge-state Ar ions are almost completely lost by axial escape while the higher charge-state Xe ions are driven out from the trap to a lesser extent. Since the rate at which energy is transferred to Ar ions is markedly reduced at lower Xe ion density, new Ar ions populate the trap and stay longer in the electron beam. The ions concentrate in the trap until their density becomes sufficiently high that the competition between Ar and Xe recommences.

\section{SAWTOOTH EXPERIMENT IN GREIFSWALD}

The EBIT was operated in a static mode, i.e. the trapping potential of the EBIT and the electron-beam energy were kept fixed during the whole measuring cycle. In adddition, the bias voltage between center and lower drift tube was fixed at $400 \mathrm{~V}$ for all measurements. After a cycle time of $25 \mathrm{~s}$ the potential at the upper drift tube was lowered to empty the trap and then the next cycle was started. During the experiment argon (nuclear charge number $Z=18)$ and xenon $(Z=54)$ were injected into the EBIT with a fixed mixing ratio (absolute gas pressure) of $6: 1$. The highest charge states for both gases were limited by the electron-beam energy of $5 \mathrm{keV}$ to $q(\mathrm{Ar})=18+$ and $q(\mathrm{Xe})=44+$. The evolution of the emitted X-rays of the trap content is shown in the scatter plot (Fig. 3 ). The detector pulse height, which is correlated to the x-ray energy, is digitized by an analog-to-digital converter and fed to a multi-channel scaler, with an energy resolution of $136 \mathrm{eV}$ and a time resolving of $50 \mathrm{~ms}$. To analyze sawtooth oscillations in the current experiment, the $\mathrm{x}$-ray emission of the $n=2-1$ transitions of $\mathrm{Ar}^{16+}$ at $3.1 \mathrm{keV}$ and $n=3-2$ transitions of $\mathrm{Xe}^{44+}$ in the range from $4.1 \mathrm{keV}$ to $4.9 \mathrm{keV}$ is used.

Several experiments were performed to investigate the effect of the absolute pressure in the gas-injection system $\left(5.5 \cdot 10^{-7} \mathrm{~Pa}\right.$ up to $\left.9 \cdot 10^{-7} \mathrm{~Pa}\right)$, the electron-beam current ( $35 \mathrm{~mA}$ up to $80 \mathrm{~mA}$ ) and the bias voltage between center and upper drift tube (-10 $\mathrm{V}$ up to $+25 \mathrm{~V})$. As described above the axial trapping potential is depending on the bias voltage between center and outer drift tubes and the image charge of the electron beam. As a consequence of the changes of the bias voltage between center and upper drift tube and the electron-beam current the axial trapping potential is varied between $0.6 \mathrm{~V}$ and 45 V. Furthermore, the axially ejected ions were detected with a Faraday cup (Fig. 4). Measurements of the energies of the ejected ions are in preparation.

\section{MODELING OF SAWTOOTH OSCILLATIONS}

The sawtooth phenomenon is very sensitive to the EBIT operating conditions and as such it is an excellent benchmark to test existing EBIT models. Since the first observation of the sawtooth oscillations, calculations of the ions time evolution were started as well 


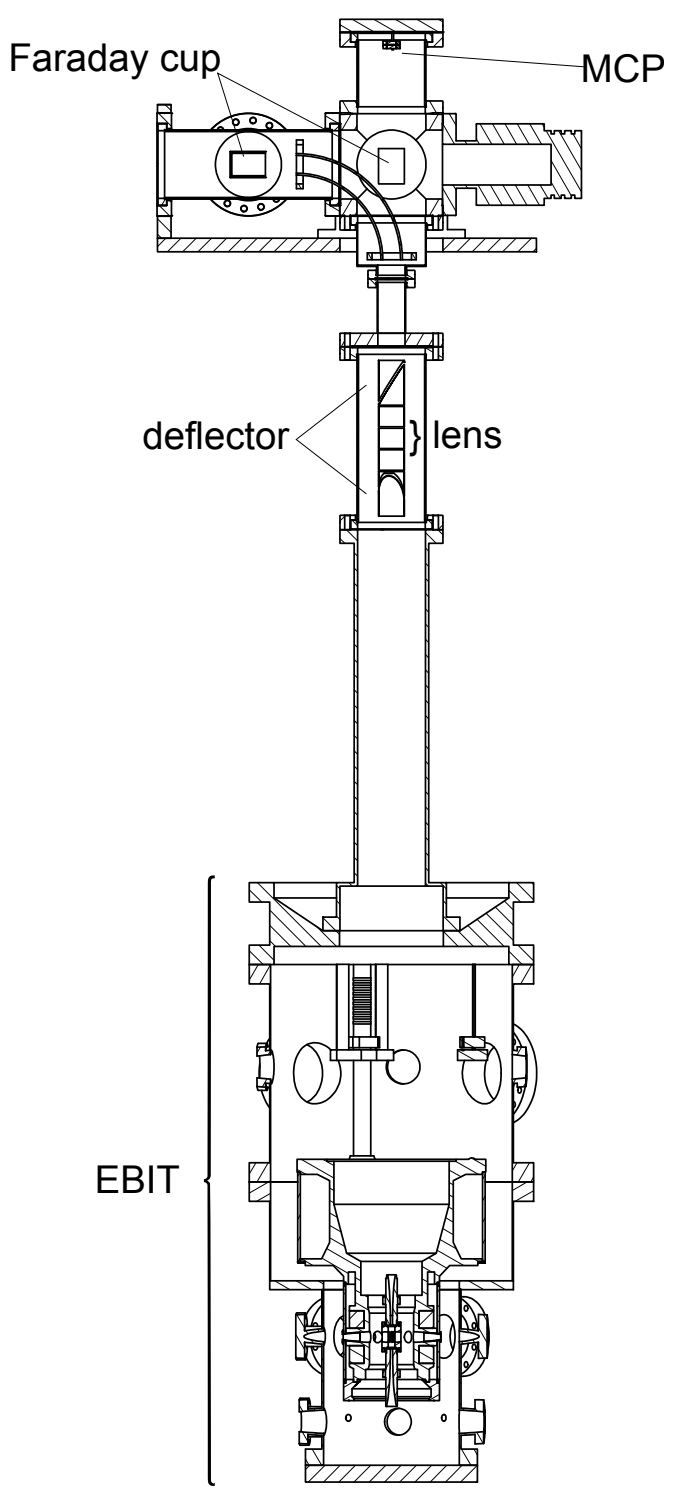

FIGURE 4. Scheme of the detection system of the Greifswald EBIT.

$[8,9,14]$. We will continue these simulations including the following processes
(a) electron-impact ionization,
(b) radiative recombination,
(c) charge exchange with neutral atoms,
(d) axial ion escape,
(e) heating of the ions by the electron beam,
(f) ion-ion energy exchange, and
(g) trap neutralization by ions. 
The time evolution of the ion density can be calculated from the following rate equation

$$
\frac{\mathrm{d} n_{\alpha, i}}{\mathrm{~d} t}=\frac{n_{\alpha, i-1}}{\tau_{\alpha, i-1}^{\mathrm{ion}}}-\frac{n_{\alpha, i}}{\tau_{\alpha, i}^{\mathrm{ion}}}+\frac{n_{\alpha, i+1}}{\tau_{\alpha, i+1}^{\mathrm{rec}}}-\frac{n_{\alpha, i}}{\tau_{i}^{\mathrm{rec}}}+\frac{n_{\alpha, i+1}}{\tau_{\alpha, i+1}^{\mathrm{ex}}}-\frac{n_{\alpha, i}}{\tau_{\alpha, i}^{\mathrm{ex}}}-\frac{n_{\alpha, i}}{\tau_{\alpha, i}^{\mathrm{esc}}}
$$

where $n_{\alpha, q}$ is the density of the trapped ions $(\alpha=\mathrm{Ar}, \mathrm{Xe})$ with charge state $i$, and $\tau_{\alpha, q}^{\text {ion }}$, $\tau_{\alpha, q}^{\mathrm{rec}}, \tau_{\alpha, q}^{\mathrm{ex}}$ and $\tau_{\alpha, q}^{\mathrm{esc}}$ are the characteristic times for creation the charge state $q$ by electronimpact ionization, for radiative recombination, for charge exchange with neutral atoms and for axial escape, respectively.

The cross section for electron-impact ionization, radiative recombination and charge exchange with neutral atoms where calculated using the expression by Lotz [15], Kim and Pratt [16], and Müller and Salzborn [17, 18], respectively. To calculate the characteristic time of the axial ion escape the expression from [19] was used. The characteristic time of the axial ion escape itself is depending on the ion temperature due to the collision rate between the ions species.

The ion temperature is calculated from the energy-balance equation including axial ion escape, heating of the ions by the electron beam and ion-ion energy exchange [9]:

$$
\frac{\mathrm{d}}{\mathrm{d} t}\left(\frac{3}{2} k T_{\alpha, i} n_{\alpha, i}\right)=-\frac{\mathrm{d} W_{\alpha, i}^{\text {esc }}}{\mathrm{d} t}+\frac{\mathrm{d} W_{\alpha, i}^{\text {beam }}}{\mathrm{d} t}+\sum_{j}\left(\frac{\mathrm{d} W_{\alpha, i}^{\text {ion-ion }}}{\mathrm{d} t}\right) .
$$

$W_{\alpha, i}^{\text {esc }}$ and $W_{\alpha, i}^{\text {beam }}$ is the energy loss by axial ion escape and the energy gain by electronbeam heating, respectively. The sum is over all Ar and Xe charge states populating the trap ( 1 to 18 for Ar and 1 to 44 for Xe) and represents the energy transfer by collisions to one ion species at charge state $i$ from all other species.

Similar trapping conditions as in the experiment were used. In all simulation an electron-beam energy of $5 \mathrm{keV}$ was used. The bias voltage between center and upper drift tube and the electron current were varied between $0 \mathrm{~V}$ up to $20 \mathrm{~V}$ and $40 \mathrm{~mA}$ up to $50 \mathrm{~mA}$, respectively. All simulations were started with neutral argon and xenon at room temperature. The density of the $\mathrm{Ar} / \mathrm{Xe}$ gas mixture was varied in the range of $10^{3} \ldots 10^{7} / \mathrm{cm}^{3}$, and the respective mixing ratio $\mathrm{Ar} / \mathrm{Xe}$ between $6: 1$ and 10 000:1. Figure 5 shows the calculated density of the argon and xenon ions. Only a few percent of the $\mathrm{Xe}^{44+}$ ions escape from the trap compared to an almost complete loss of the $\mathrm{Ar}^{16+}$ population. As described above, the ion escape is correlated with an increase of the ion temperature.

\section{CONCLUSIONS AND OUTLOOK}

After reinstallation of the former Berlin EBIT in Greifswald sawtooth oscillations of $\mathrm{Ar} / \mathrm{Xe}$ mixtures were investigated by x-ray measurements. In addition, it was possible to observe the axially ejected ions with a Faraday cup. In a next step, their energies will be measured using a combination of a grid at variable retarding potential and a multichannel plate detector (see Fig. 4). In addition, calculations of the sawtooth oscillations were performed. We could reproduce the ion escape from the trap. However, the calculated time interval between successive ion expulsions differs from the experiments. Obviously, it is necessary to improve the present EBIT simulation. 


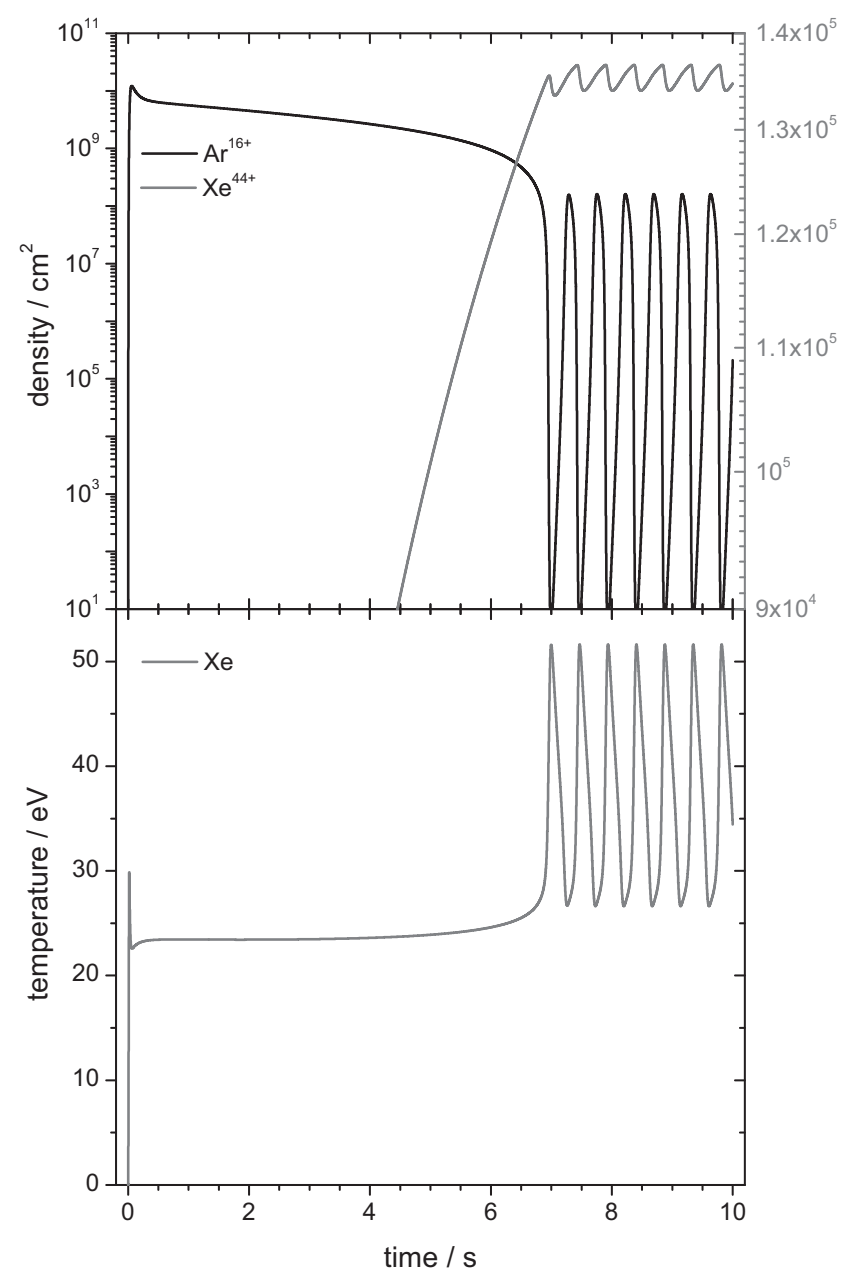

FIGURE 5. (Top) Simulations of swatooth oscillations at a gas-mixing ratio of $\mathrm{Xe}: \mathrm{Ar}=1: 1000$, an electron current of $40 \mathrm{~mA}$ at an energy of $5 \mathrm{keV}$ and a bias voltage of $10 \mathrm{~V}$. (Bottom) Temperature of the $\mathrm{Xe}^{44+}$ ions, which is similar to the temperature of the $\mathrm{Ar}^{16+}$ ions.

In parallel, a beamline for collision experiments between HCI and fullerenes was constructed. First measurements with gas targets are in preparation. Test experiments with fullerenes are scheduled, too. These will be followed by metal cluster investigations.

\section{ACKNOWLEDGMENTS}

The project is funded by the Max Planck Foundation. We thank the Max Planck Institute of Plasma Physics for providing the former Berlin EBIT. 


\section{REFERENCES}

1. C. Biedermann, T. Fuchs, G. Fußmann, and R. Radtke, "The Berlin Electron Beam Ion Trap," in Trapping highly charged ions: Fundamentals and applications, edited by J. Gillaspy, Nova Science Pub Inc., 2001, chap. 2, pp. 81-101.

2. F. Currell, and G. Fussmann, IEEE Transactions on Plasma Science 33, 1763-1777 (2005).

3. M. Levine, R. Marrs, J. Henderson, D. Knapp, and M. Schneider, Physica Scripta T22, 157 (1988).

4. R. E. Marrs, S. R. Elliott, and D. A. Knapp, Phys. Rev. Lett. 72, 4082-4085 (1994).

5. H. Cederquist, A. Fardi, K. Haghighat, A. Langereis, H. T. Schmidt, S. H. Schwartz, J. C. Levin, I. A. Sellin, H. Lebius, B. Huber, M. O. Larsson, and P. Hvelplund, Phys. Rev. A 61, 022712 (2000).

6. A. Reinköster, B. Siegmann, U. Werner, B. A. Huber, and H. O. Lutz, Journal of Physics B: Atomic, Molecular and Optical Physics 35, 4989 (2002).

7. J. Jin, H. Khemliche, M. H. Prior, and Z. Xie, Phys. Rev. A 53, 615-618 (1996).

8. R. Radtke, and C. Biedermann, Phys. Rev. A 67, 032705 (2003).

9. R. Radtke, C. Biedermann, P. Bachmann, G. Fussmann, and T. Windisch, Journal of Physics: Conference Series 2, 84 (2004).

10. Y. Fu, D. Lu, B. Wei, Y. Yang, Y. Liu, W. Chen, K. Yao, J. Xiao, Y. Shen, M. He, W. Hu, X. Zhang, B. Zhang, L. Liljeby, and Y. Zou, Physica Scripta T134, 014017 (2009).

11. C. Biedermann, A. Förster, G. Fußmann, and R. Radtke, Physica Scripta T73, 360 (1997).

12. M. Levine, R. Marrs, J. Bardsley, P. Beiersdorfer, C. Bennett, M. Chen, T. Cowan, D. Dietrich, J. Henderson, D. Knapp, A. Osterheld, B. Penetrante, M. Schneider, and J. Scofield, Nuclear Instruments and Methods in Physics Research Section B: Beam Interactions with Materials and Atoms 43, 431 440 (1989).

13. M. A. Levine, R. E. Marrs, J. R. Henderson, D. A. Knapp, and M. B. Schneider, Physica Scripta T22, 157 (1988).

14. R. Radtke, C. Biedermann, and P. Bachmann, Nuclear Instruments and Methods in Physics Research Section B: Beam Interactions with Materials and Atoms 205, 250 - 254 (2003).

15. W. Lotz, Zeitschrift für Physik A Hadrons and Nuclei 206, 205-211 (1967).

16. Y. Kim, and R. Pratt, Physical Review A 27, 2913 (1983).

17. B. M. Penetrante, J. N. Bardsley, D. DeWitt, M. Clark, and D. Schneider, Phys. Rev. A 43, 4861-4872 (1991).

18. A. Müller, and E. Salzborn, Physics Letters A 62, 391-394 (1977).

19. G. Fussmann, C. Biedermann, and R. Radtke, "EBIT: An electron beam source for the production and confinement of highly ionized atoms," in ADVANCED TECHNOLOGIES BASED ON WAVE AND BEAM GENERATED PLASMAS, edited by H. Schluter, and A. Shivarova, SPRINGER, 1999, vol. 67 of NATO ADVANCED SCIENCE INSTITUTE SERIES, SUB-SERIES 3, HIGH TECHNOLOGY, pp. $429-468$. 